\title{
QUISTE DERMOIDE EN REGIÓN LATERAL DE CUELLO. PRESENTACIÓN DE UN CASO Y REVISIÓN DE LA LITERATURA
}

Dermoid cyst in the lateral neck region. Presentation of a case and review of the literature

Fecha de Recepción: 25 de enero 2021
Cisto dermóide na região lateral do pescoço. Relato de caso e revisão da literatura

Aceptado para su publicación: 17 de marzo 2021

\author{
Autores: \\ Mauricio González Rebattú y González ${ }^{1, a}$ \\ Ana María Nieto Munguía ${ }^{1, a}$ \\ José Manuel González Avilés ${ }^{1, b}$ \\ Jesús Lorenzo Derat Araujo ${ }^{1, c}$ \\ Emmanuel Hernández Pérez ${ }^{1, d}$
}

1. Servicio de Cirugía Maxilofacial. Hospital Regional " $1{ }^{\circ}$ de Octubre". Instituto de Seguridad y Servicios Sociales de los Trabajadores del Estado (ISSSTE). Ciudad de México, México. a. Especialista en Cirugía Oral y Maxilofacial (Universidad Nacional Autónoma de México).

b. Especialista en Oncología Quirúrgica (Universidad Nacional Autónoma de México). c. Cirujano dentista (Centro de Estudios Universitarios Superiores. Culiacán, Sinaloa México). d. Cirujano dentista (Universidad Nacional Autónoma de México).

Correspondencia: González Rebattú y González, Mauricio Hospital Regional $1^{\circ}$ de Octubre, ISSSTE Servicio de Cirugía Maxilofacial, Consulta Externa. Av. Instituto Politécnico Nacional No 1669 Magdalena de las Salinas, Gustavo A. Madero, México, D.F. + 525555866011

Correo electrónico: mauriciorebattu@yahoo.com.mx

Conflicto de intereses: los autores declaran no tener conflictos de interés.

Fuente de financiamiento: autofinanciado.

\section{Resumen}

Los quistes dermoides verdaderos son entidades congénitas raras con predilección por las áreas periorbitarias, piso de boca y submentonianas. En la región de cabeza y cuello la frecuencia es baja (menos de $2 \%$ ). El quiste se presenta como una masa blanda, indolora y desplazable. La mayoría son pequeños, pero algunos superan los $12 \mathrm{~cm}$. Por lo general, son asintomáticos a menos que se agranden sustancialmente, causando dolor o dificultades funcionales. La enucleación completa es el tratamiento de elección. El objetivo de este trabajo fue presentar el caso clínico de un paciente femenino de 52 años de edad con aumento de volumen en región cervical de lado izquierdo, con una evolución de dos años. Fue tratado mediante enucleación, con un abordaje extraoral submandibular tipo Risdon y presentó una evolución favorable.

Palabras clave: quiste dermoide; cuello; cirugía maxilofacial (fuente: DeCS BIREME).

\begin{abstract}
True dermoid cysts are rare congenital entities with predilection for the periorbital, floor of the mouth and submental areas. In the head and neck region the frequency is low (less than 2\%). The cyst appears as a soft, painless and movable mass. Most of them are small, but some may be larger than $12 \mathrm{~cm}$. They are usually asymptomatic unless they enlarge substantially, causing pain or functional difficulties. Complete enucleation is the treatment of choice. The aim of this work was to present the clinical case of a 52-year-old female patient with enlargement in the cervical region on the left side, with an evolution of two years. She was treated by enucleation, with a Risdon type submandibular extraoral approach and presented a favorable evolution.
\end{abstract}


Key words: dermoid cyst; neck; maxillofacial surgery (source: MeSH NLM).

\section{Resumo}

Os cistos dermóides verdadeiros são entidades congênitas raras com predileção pela região periorbital, assoalho da boca e áreas submentais. Na região da cabeça e pescoço a frequência é baixa (menos de $2 \%$ ). O cisto se apresenta como uma massa macia, indolor e deslocável. A maioria é pequena, mas alguns ultrapassam os $12 \mathrm{~cm}$. Geralmente são assintomáticos, a menos que estejam substancialmente aumentados, causando dor ou dificuldades funcionais. A enucleação completa é o tratamento de escolha. O objetivo deste trabalho foi apresentar o caso clínico de uma paciente de 52 anos com aumento de volume na região cervical à esquerda, com evolução de dois anos. Foi tratado por enucleação, com acesso extraoral submandibular tipo Risdon e apresentou evolução favorável.

Palavras-chave: cisto dermoide; pescoço; cirurgia maxilofacial (fonte: DeCS BIREME).

\section{Introducción}

Los quistes dermoides (QD) son neoplasias de comportamiento benigno que pueden presentarse desde el nacimiento, de crecimiento lento, pero localmente invasivas derivadas de células con la habilidad de diferenciarse de cualquiera de los tejidos procedentes de las tres capas germinales ${ }^{1,2}$. Meyer realizó una clasificación de acuerdo a los derivados embrionarios que contienen, los agrupó en: 1) epidermoides, quistes revestidos de epitelio, pero sin estructuras anexas, 2) dermoides, quistes con anexos dérmicos, 3) teratoides, quistes revestidos de epitelio que contienen elementos epiteliales y no epiteliales como hueso, músculo, tejido respiratorio o gastrointestinal y 4) teratomas verdaderos ${ }^{3}$.

Otra clasificación distinguió tres tipos diferentes según la relación anatómica entre el QD y los músculos del piso de la boca: 1) quistes genioglosos o sublinguales medianos, por encima del músculo geniohioideo; 2) quistes geniohioideos o submentonianos medianos, entre los músculos geniohioideo y milohioideo; 3) quistes laterales debajo del cuerpo de la mandíbula. Los quistes laterales son los más raros y, a menudo, desplazan las estructuras anatómicas circundantes (por ejemplo, la glándula submandibular y su conducto). Algunos autores no creen que existan quistes laterales. Consideran que se originan en la posición media y luego se expanden lateralmente, la persistencia de la parte media prueba el origen de la línea media ${ }^{4}$.

La etiología de estos quistes se atribuye al atrapamiento de detritus epitelial en la línea media durante la fusión del primer y segundo arco faríngeo, durante la quinta semana de desarrollo embrionario. Su incidencia oscila entre 0.05 y $2 \%$, sin predilección por sexo, $7 \%$ se presenta en la cabeza o cuello, varían de unos pocos milímetros a $12 \mathrm{~cm}$ de diámetro. Son raros en el cuello, pero pueden representar cerca de un cuarto $(22 \%)$ de las lesiones del cuello en la línea media o casi en la línea media. Se han reportado en la parte superior del cuello, cerca del cartílago tiroides y tan bajo como la muesca supraesternal ${ }^{1,5}$.

A la palpación son blandos y pastosos debido a la queratina y al sebo que contienen en su luz, además de formaciones o de anexos cutáneos como folículos pilosos, glándulas sudoríparas o glándulas sebáceas y/o algunas veces dientes. La palpación bimanual es suficiente para detectar un QD ya sea superficial o profundo al músculo geniohioideo. Si es superficial, es posible notar una distensión de los tejidos blandos de la región submentoniana. Otro signo patognomónico es un aumento de la tensión cervical provocada por el quiste, que produce la contracción de los músculos milohioideo y geniohioideo. La luz del quiste generalmente contiene una mezcla de queratina descamada, sebo y tallos pilosos. La cápsula del quiste está formada por una estrecha zona de tejido conectivo comprimido que está generalmente exento de inflamación. Con frecuencia distienden la mucosa tan a fondo que su contenido puede ser visible $^{4,6}$.

Una vez identificado, la localización del quiste determina el enfoque de la escisión. Según su localización se dividen en mediales y laterales, aunque algunos autores afirman que la variedad lateral no es más que un crecimiento del quiste desde la línea media. Suelen ser masas de consistencia laxa que aumentan de tamaño gradualmente. No fluctúan al tacto y a menudo se encuentran adheridas al hueso hioides ${ }^{5}$. 


\section{REFO}

VOL. XIV | N 1| AÑO 2021

ISSN 1668-7280

ISSN-E 2683-7986
Los QD se tratan mediante extirpación quirúrgica. Los ubicados por encima del músculo genihioideo pueden retirarse mediante una incisión intraoral y aquéllos por debajo del músculo genihioideo pueden requerir un enfoque extraoral. Con uno u otro enfoque, la eliminación es difícil debido a la adherencia del quiste a los tejidos circundantes $y$ al tamaño que pudieran alcanzar. Tienden a estar fibrosados a los tejidos circundantes, lo que requiere una disección roma más aguda. La transformación maligna en carcinoma de células escamosas se ha reportado en raras ocasiones. La recurrencia es poco común teniendo un pronóstico favorable6.

\section{Caso clínico}

Presentamos el caso de un paciente femenino de 52 años de edad, sin antecedentes crónico degenerativos ni quirúrgicos de importancia, la cual comienza con padecimiento actual hace dos años al presentar aumento de volumen en región cervical de lado izquierdo, motivo por el cual acude a su Unidad de Medicina Familiar (UMF) donde le realizan estudios de gabinete sin obtener diagnóstico alguno, por lo que es referida al servicio de cirugía maxilofacial del Hospital Regional 1ro de octubre (ISSSTE) para su valoración.

A la exploración física se observó un aumento de volumen en región cervical lateral de lado izquierdo, de aproximadamente $5 \times 5 \mathrm{~cm}$ de longitud, asintomática, bien delimitada, de consistencia blanda, de coloración normal al tejido adyacente y no adherida a planos profundos (Figura 1).

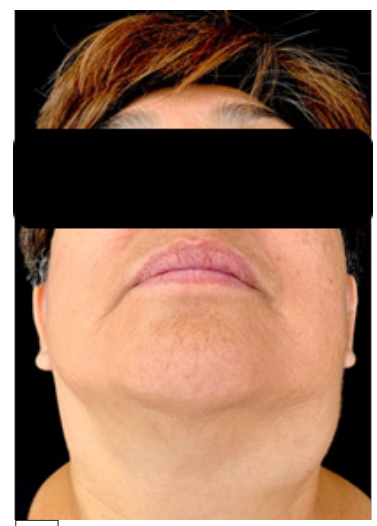

A

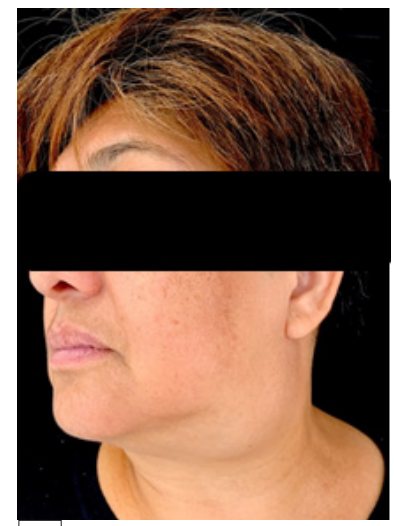

B
Figura 1. (A) imagen extraoral cefalo-caudal. Se observa aumento de volumen en región cervical lateral de cuello de lado izquierdo. (B) imagen extraoral en tres cuartos. Se observa aumento de volumen en región cervical lateral de cuello de lado izquierdo.
El estudio mediante tomografía computarizada de macizo facial con extensión a cuello en fase simple y contrastada desde base de cráneo hasta región supraesternal muestra una imagen hipodensa de bordes definidos, en zona 2 y nivel 2 ganglionar de cuello. Se extiende en región submentoniana y en sentido contralateral de lado izquierdo. Presenta densidad heterogénea que va desde contenido graso a solido menor a $30 \mathrm{UH}$, sin mostrar reforzamiento con aplicación de medio de contraste ni tampoco muestra de realce periférico (Figura 2).
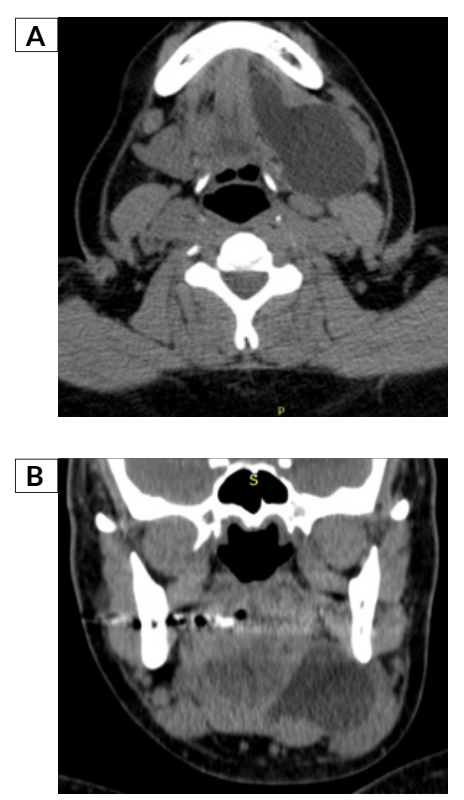

Figura 2. (A) tomografía computarizada contrastada en un corte axial y $(B)$ corte sagital en las cuales se observa área hipodensa a tejidos blandos bien delimitada, con discreto desplazamiento de músculos genihoideos.

Se realizó procedimiento quirúrgico bajo anestesia general balanceada, mediante abordaje transfacial submandibular extraoral tipo Risdon7. Mediante disección roma se logró su completa enucleación, se colocaron puntos de sutura en planos profundos con vicryl 3-0, así como dren tipo Penrose y se suturó piel con puntos subdérmicos con nylon 5-0 (Figura 3).

El espécimen quirúrgico obtenido se fijó en formol amortiguado al $10 \%$ durante 24 horas y posteriormente fue enviado para su estudio histopatológico. Macroscópicamente se trató de un espécimen de tejido blando de $6 \times 3 \mathrm{~cm}$ de longitud de coloración café y amarilla de consistencia pastosa, superficie lisa y rugosa (Figura 3). 


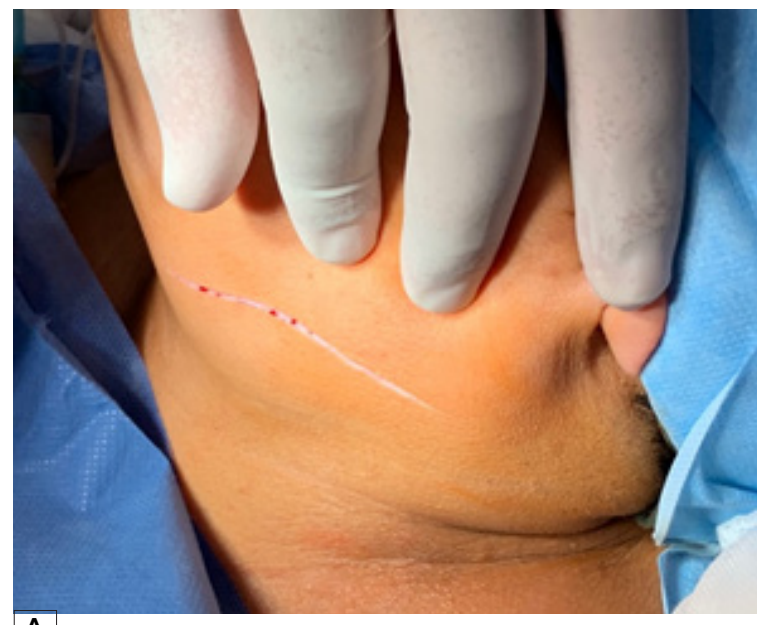

A

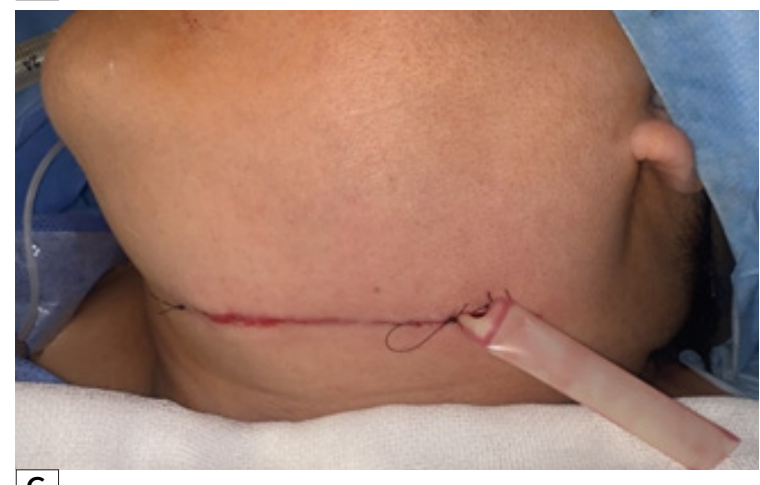

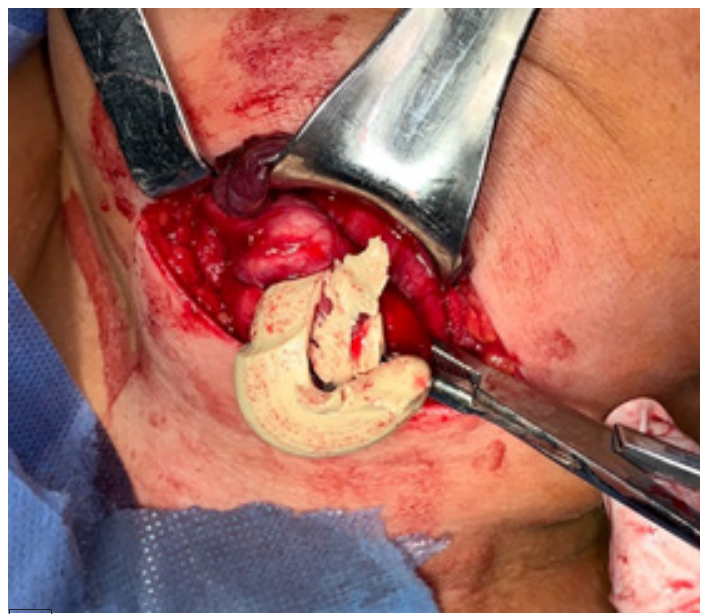

B

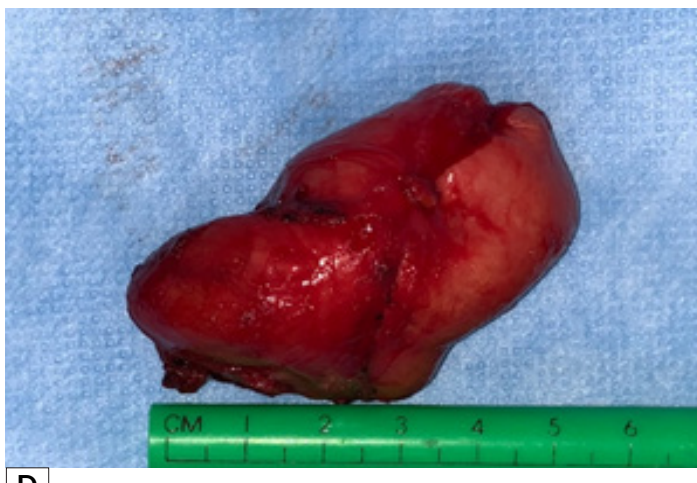

D

Figura 3. (A) incisión transfacial submandibular tipo Risdon, (B) disección de la lesión quística, (C) sutura subdérmica con nylon 5-0 con colocación de dren tipo Penrose y postquirúrgico inmediato. (D) espécimen quirúrgico inmediato de superficie lisa de $5 \times 3 \mathrm{~cm}$.

En los cortes histológicos examinados se observó una cavidad quística llena de queratina fibrilar por epitelio escamoso estratificado hiperortoqueratinizado. La cápsula es de tejido conectivo fibroso denso, bien vascularizado con glándulas sebáceas e infiltrado inflamatorio crónico severo difuso, con células gigantes multinucleadas a cuerpo extraño, tejido adiposo maduro y hemorragia reciente (Figura 4).

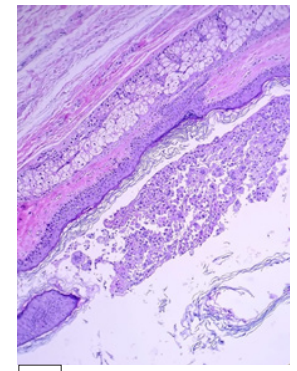

A

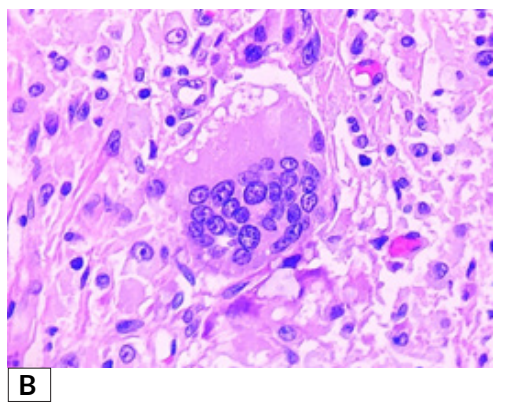

Figura 4. Figura 4: Hematoxilina-eosina (A) magnificación 10x, se observa una cavidad quística revestida por epitelio plano estratificado queratinizado hacia la luz con abúndate queratina. (B) magnificación 40x, en la capsula de tejido conectivo fibroso denso se aprecian glándulas sebáceas, vasos de diferentes calibres, con células gigantes multinucleadas a cuerpo extraño.

\section{Discusión}

Los principales diagnósticos diferenciales incluyen: el quiste del conducto tirogloso, el cual se presenta clásicamente en la línea media cervical como un aumento de volumen asintomático, móvil y fluctuante desde el agujero ciego hasta la horquilla esternal. El quiste del segundo arco branquial por lo general muestra aumento de volumen laterocervical, no doloroso, y fijo a planos profundos. Los abscesos sublinguales y celulitis sublingual, ambos procesos infecciosos, que clínicamente pueden mostrar aumento de volumen, doloroso y fluctuante. La ránula clínicamente presenta aumento de volumen sublingual unilateral y puede crecer hasta desplazar la lengua hacia atrás interfiriendo en la masticación y el habla, la mucosa superficial de la lesión con frecuencia es muy delgada adquiriendo un color blanco-azulado. El higroma quístico se trata de una anomalía congénita de los vasos linfáticos que impide el correcto drenaje de la linfa, es de consistencia blanda y fluctuante, con contenido líquido, su localización en orden de frecuencia es cervical, axilar e inguinal ${ }^{8-11}$. Estos diagnósticos diferenciales, 


\section{CASO CLÍNICO}

\section{REFO}

VOL. XIV | No 1| AÑO 2021

ISSN 1668-7280

ISSN-E 2683-7986 en nuestro caso clínico fueron descartados en base a las características clínicas y de imagen por tomografía computarizada en fase simple y constrastada.

Por lo general, los QD se desarrollan con mayor frecuencia sobre el músculo milohioideo, situados en la línea media del triángulo sublingual o piso de la boca ${ }^{12}$. En la presentación de nuestro caso clínico, la localización anatómica del quiste se desarrolló por debajo del músculo milohioideo de manera cervical lateral de lado izquierdo que involucró la zona 2 y nivel 2 ganglionar de cuello.

Aunque generalmente se diagnostican en la segunda y tercera décadas de la vida, pueden presentarse a cualquier edad con la misma frecuencia de aparición en ambos sexos. Dependiendo del tamaño de la lesión, puede desplazar la lengua y causar disfagia, disfonía y disnea ${ }^{11}$. En este caso clínico, se presentó un quiste dermoide en región lateral de cuello izquierdo, en un paciente femenino de la sexta década de la vida quien busco atención en nuestro servicio por presentar dificultades principalmente a la deglución, así como también por razones estéticas.

La tomografía computarizada en fase contrastada se ha convertido en el estudio de elección para el diagnóstico, localización y selección del tratamiento quirúrgico a elegir para el QD, si bien se pueden utilizar otras modalidades de estudios de gabinete como la ultrasonografía de alta resolución y la resonancia magnética, el estándar de oro sigue siendo la tomografía computarizada ${ }^{13}$.

El abordaje submandibular tipo Risdon representa una técnica adecuada para la localización de QDs desarrollados por debajo del músculo milohioideo, los pacientes presentan una tasa baja de complicaciones, así también la adecuada visibilidad del abordaje permite realizar una disección roma cuidadosa de la lesión disminuyendo la tasa de recidiva ${ }^{14}$.

La evaluación de la localización exacta mediante examen clínico, aspiración parcial e inyección de medios de contraste con radiografía, tomografía computarizada o resonancia magnética es esencial antes de planificar el abordaje quirúrgico ${ }^{13}$.
El tratamiento consiste en la extirpación quirúrgica completa mediante disección meticulosa y no romper el quiste, ya que el contenido luminal puede actuar como irritante para los tejidos fibrovasculares, produciendo edema postoperatorio. La recurrencia es poco probable después de la escisión quirúrgica completa de la cápsula fibrosa de estas lesiones, pero debe considerarse la posibilidad de transformación maligna de QDs en tipo teratoide ${ }^{11}$. El abordaje quirúrgico está determinado por la relación del quiste con la musculatura, en los quistes superiores al músculo milohioideo se prefiere el abordaje intraoral, mientras que las lesiones inferiores se extirpan por abordaje extraoral ${ }^{14}$.

En el presente caso se observó un aumento de volumen en región lateral de cuello de lado izquierdo por lo que de acuerdo a los hallazgos clínicos y con base en literatura encontrada en esta revisión, se decidió realizar un abordaje submandibular tipo Risdon, el cual proporciona un método seguro, rápido y efectivo que puede conducir a la fácil enucleación de un quiste dermoide en región submandibular, ya que dicha técnica es la más beneficiosa para el paciente de acuerdo a los criterios de localización y clasificación de QDs en región de cabeza y cuello. 


\section{Referencias Bibliográficas}

1. Alonso Moctezuma A, Salgado Chavarría F, Zeta Castañeda E, Robles Calzada L, Portilla Robertson J, Molotla Fragoso A. Quiste teratoide congénito en piso de boca. Presentación de un caso y revisión de la literatura. Rev Mex Cir Bucal Maxilofac 2020;16 (1):36-41. doi: $10.35366 / 93386$

2. Jayasuriya NSS, Siriwardena S, Tilakaratne WM, Parthiepan S. Malignant transformation of a long-standing submental dermoid cyst to a carcinosarcoma: a case report. J Med Case Reports. 2017;11(11). doi: 10.1186/ s13256-016-1186-y

3. Meyer I. Dermoid cysts (dermoids) of the floor of the mouth. Oral Surg Oral Med Oral Pathol. 1955;8(11):114964. doi: 10.1016/0030-4220(55)90380-7

4. Brusati R, Galioto S, Tullio A, Moscato G. The Midline Sagittal Glossotomy for Treatment of Dermoid Cyst of the Mouth Floor. J Oral Maxillofa Surg. 1991;49:875-878.

5. Gomez-Carrillo V, Martinez-Seijas P, Diaz-Galvis L, Martin J, Rad J, Esnal F et al. Técnica Quirúrgica para abordaje intraoral de quiste dermoide cervical de gran tamaño: a proposito de un caso clinico. Rev Esp Cir Oral Maxilofa. 2011;33(3):124-128. doi:10.1016/j.maxilo. 2011.04.001 6. González de Santiago M, Alatorre Pérez S, Montaño SA, Ayala Bernal JM. Quiste Dermoide. Reporte de dos casos clínicos. Rev Mex Cir Bucal Maxilofac. 2014;11(1):20-26. Disponible en: https://www.medigraphic.com/pdfs/cirugiabucal/cb-2015/cb151e.pdf
7. Contreras Alvarado EA, Theriot Girón MC, Quiroga García MA, Hernández Juárez J. Estructuras anatómicas de riesgo en el abordaje submandibular. Rev Mex Estomatol. 2017;7(1):48-56. Disponible en: https://www.remexesto. com/index.php/remexesto/article/view/149/272

8. Pentenero M, Marino R, Familiari U, Gandolfo S. Choristoma involving the floor of the mouth and the anterior tongue: a case of teratoid cyst with gastric and respiratory epithelia. J Oral Maxillofac Surg. 2013;71(10):1706-1711. doi: 10.1016/j.joms. 2013.04.020

9. He J, He Y, Zhu H, Wang Y, Qiu W. Congenital huge submandibular and neck teratoid cyst in newborn. J Craniofacl Surg. 2015;26(2):565-567. doi: 10.1097/ SCS.0000000000001278

10. Palaskar SJ, Garde J, Narang B. Teratoid cyst of the oral cavity: a rare entity. J Oral Maxillofac Pathol. 2014; 18 (3): 469-471. doi:10.4103/0973-029X.151359

11. Vieira EM, Borges AH, Volpato LE, Porto AN, Carvalhosa AA, Botelho Gde A, Bandeca MC. Unusual dermoid cyst in oral cavity. Case Rep Pathol. 2014;2014:389752. doi: 10.1155/2014/389752

12. Tandon PN, Gupta DS. Epidermoid cyst in the floor of mouth with submental component. J Maxillofac Oral Surg. 2014;13(1):59-62. doi:10.1007/s12663-010-0098-4

13. Ariyoshi Y, Shimahara M. Magnetic Resonance Imaging of a Submental Dermoid Cyst: Report of a Case. J Oral Maxillofac Surg. 2003;61(4):507-510. doi:10.1053/ joms.2003.50097

14. Mandel L, Surattanont F. Lateral dermoid Cyst. J Oral Maxillofac Surg. 2005;63(1):140-144. doi: 10.1016/j. joms. 2004.04.028 